\title{
Des traces indélébiles de traumas cachés
}

\author{
Marion Feldman ${ }^{1}$
}

\section{Résumé}

Cet article questionne la réactivation de traumatismes anciens à l'aune d'une actualité sociale effractante. Il s'agit ici du vécu des enfants juifs cachés en France pendant la Seconde Guerre mondiale et de la façon dont ils se sont construits tout au long de leur vie, en identifiant les réaménagements psychiques inhérents à des âges passages: adolescence, parentalité, grand-parentalité, retraite. Trois illustrations cliniques montrent comment le passage à la retraite pour un patient, l'approche de la mort pour une patiente et les attaques meurtrières en France de janvier et novembre 2015 pour une autre, viennent réactiver ce qui n’a jamais pu être élaboré à un temps très ancien, et comment une élaboration dans l'après-coup s'avère indispensable, même à un âge avancé de la vie.

P our échapper à la déportation qui menaçait tous les Juifs d'Europe pendant la Seconde Guerre mondiale, un certain nombre d'enfants ont été contraints de vivre cachés pour assurer leur survie. Ils pouvaient devenir invisibles ou rester visibles (Dwork, 1991), c'est-à-dire qu'ils étaient soit cachés au sens propre du terme, dans un endroit clos pour demeurer invisibles physiquement, soit cachés avec un changement d'identité, de prénom, de nom, de religion, d'environnement et, pour la plupart d'entre eux, séparés de leurs parents. Ces enfants ont ainsi été exposés à des attaques des liens de filiation, des liens d'affiliation, des pertes, des privations, parfois des humiliations et des maltraitances, et des frayeurs multiples (Feldman, 2009). Outre ces traumas, ils ont dû supporter le silence et l'absence de reconnaissance de leur vécu. Enfants, ils se sont construits de façon spécifique, en lien avec cette situation singulière d'avoir été cachés. Ils devaient notamment taire leur identité. De plus, la Libération n'en a pas été une pour eux, et il a fallu attendre l'année 1991 pour que la souffrance des «enfants cachés» soit reconnue officiellement.

Ils ont grandi avec le silence de la période de clandestinité et celui de l'après-guerre. Ils ont ainsi été doublement «silenciés » (Cherki, 2006).
Ces traumatismes liés à ce vécu ont eux-mêmes souvent été cachés, non divulgués, non partagés avec la famille: conjoint, enfants, petits-enfants, notamment par le fait que la reconnaissance de leur souffrance a été tardive (Feldman, 2016). Ces traumatismes «cumulatifs» (Kahn, 1976) qui ont fait effraction dans le passé infantile se manifestent, à certains moments précis de la vie psychique également en lien avec une réalité sociale.

\section{Des traces des traumas, visibles à certaines périodes de vie}

Les traces de l'impact traumatique se sont révélées être particulièrement repérables à des âges clés de la vie (Feldman et al., 2008; Feldman et Hammami, 2017) : adolescence, maternité, parentalité, retraite, vieillesse, grand-parentalité, dernier cycle de vie... Ces âges constituent des passages qui, le plus souvent, sont des périodes de réaménagements

Professeure de psychopathologie psychanalytique, Université Paris Nanterre; chercheure au laboratoire Clinique, Psychanalyse, Développement CLIPSYD EA4430 (A2P), psychologue-clinicienne. 
psychiques, fragilisant l'individu, ou pouvant être, au contraire, des leviers de transformation.

Dans l'après-guerre, l'adolescence a été une période traversée par des questions importantes autour des appartenances «affiliatoires» et de leur propre «survivance» (Altounian, 2000), accompagnée d'un sentiment de culpabilité: «Suis-je juif et/ ou catholique?», «Suis-je juif et/ou protestant?», «Quelle légitimité à être?», «Pourquoi ai-je survécu et pas les autres?». Les questions autour des identifications secondaires se sont posées pour nombre d'entre eux. Pour certains, cela a également été un moment d'engagement dans des mouvements de jeunesse, souvent sous l'impulsion de leurs parents ou oncles et tantes. Cela a également constitué une période de voyages vers Israël, pays récemment créé. Les recherches de solutions ont été nombreuses pour tenter de répondre à ce grand malaise à être.

À la suite de la période pubertaire, le mariage a souvent été précipité, notamment pour les femmes. Quand elles ont retrouvé leurs parents ou un des deux parents après la guerre, il s'est souvent avéré que les retrouvailles ont été difficiles. Le mariage a alors été une issue pour échapper à l'emprise parentale, difficile à vivre. Dans le même temps, il fallait s'autonomiser et devenir indépendant. Il en a été de même pour les orphelins, qui ont grandi, après la guerre, pour nombre d'entre eux, en maisons d'enfants (Hazan, 2000). Le mariage a constitué une issue pour ne plus être à la charge des organisations juives qui en étaient responsables. Les femmes sont parties dès leur majorité, à 21 ans, souvent pour se marier. Il s'agissait le plus souvent d'un mariage avec un conjoint juif ashkénaze quand les parents étaient présents et qu'ils pouvaient encore imposer une autorité sur leurs enfants. D'autres se sont mariés soit avec des Juifs sépharades, soit avec des catholiques. Les configurations ont été différentes. De façon générale, les mariages et la vie conjugale se sont avérés compliqués.

Pour beaucoup de femmes, la maternité a souvent été accompagnée d'une grande difficulté à se sentir mère (Feldman et al., 2013). Après le parcours chaotique, avec notamment une discontinuité de la relation avec l'objet primaire, il a semblé difficile pour ces femmes d'avoir une «préoccupation maternelle primaire» (Winnicott, 1956) qui s'inscrivait dans la continuité. Parfois, elles ont rejoué ce qu'elles avaient vécu elles-mêmes, en confiant leur enfant à une nourrice, ou à leur propre mère qui s'est quelquefois approprié l'enfant comme étant le sien, ou bien encore elles ont été dans une surprotection maternelle, percevant l'extérieur comme menaçant. La fluctuation ou l'absence de modèle parental, l'instabilité dans leur relation à l'objet primaire se sont trouvées réactivées dans leur nouveau statut parental.

C'est souvent à l'adolescence de leurs propres enfants que les parents se sont rendu compte d'un malaise familial, via des troubles que pouvaient présenter leurs enfants: troubles du comportement alimentaire, bouffées délirantes aiguës, tentatives de suicide... Et c'est à ce moment-là que certains d'entre eux ont commencé une démarche en psychothérapie - même s'il s'agit d'une minorité dans la population rencontrée.

La reconnaissance sociale et politique de leur souffrance a eu un impact considérable. 1991 a été une date importante, par la tenue de la première réunion internationale des «enfants cachés» qui a eu lieu à New York. En France, 1995 est la date du discours du Président Jacques Chirac reconnaissant la responsabilité de la France dans les crimes commis envers les Juifs pendant l'Occupation.

Cependant, le temps de la retraite a parfois été le temps d'une potentielle décompensation. Des malaises se sont fait ressentir à cette période de la vie: l'arrêt du travail a réactivé des pertes, des séparations anciennes. La venue des petits-enfants a permis, dans certains cas, de faire advenir de la circularité. Cependant, les relations avec leurs enfants se sont avérées particulièrement complexes et le lien avec la descendance s'en est trouvé/s'en trouve parfois empêché. L'entrée en maison de retraite réactive parfois des séparations anciennes et douloureuses.

En outre, entrant dans leur dernier cycle de vie et avec les difficultés que suscite la vieillesse, des angoisses et des inquiétudes surviennent, et ces dernières sont d'autant plus fortes qu'il s'agit de personnes ayant eu un passé infantile traumatique.

De façon générale, cette phase de vie correspond à des changements de vie importants tels que: l'éloignement des enfants, la mort d'amis, l'entrée de certains proches en institution. Ces éléments peuvent constituer un rappel de traumatismes qui réactivent la douleur de la perte de familles entières et des séparations irréversibles. Les sentiments habituellement éprouvés par les personnes 
âgées - sentiments d'abandon, d'isolement, de séparation - sont ici ranimés, puisque ces mêmes sentiments ont déjà été vécus dans des situations traumatiques, soixante-dix ans auparavant.

\section{Une boîte «a-larme(s)»}

Un jour, un homme âgé de 70 ans me demande un rendez-vous. Monsieur T. est né fin 1940. C'est sa femme qui l'accompagne à son premier rendez-vous au cours de l'année 2010. Madame T. a lu mes articles et a vivement conseillé à son mari de venir me voir pour être aidé. Madame est un support d'étayage important pour son mari. Monsieur T. me dit que depuis qu'il est à la retraite, il ne cesse de pleurer. Il ne comprend pas pourquoi car avant il ne pleurait jamais.

Il viendra seul à partir du deuxième rendez-vous. Monsieur T. me parle de son enfance. Début 1943, sa mère et lui sont arrêtés par la gestapo. Ils sont emmenés au camp de Drancy. À ce moment-là, il ne sait pas quel concours de circonstances a fait que lui est libéré, mais pas sa mère. Son père arrive à le récupérer et le met à l'abri dans différentes familles qui se succéderont. Il ne reverra pas sa mère qui a été déportée à Auschwitz.

Monsieur T. a bien réussi professionnellement, il était ingénieur. Il s'est marié avec une femme catholique et ils ont eu trois enfants. Sa femme a tenu à baptiser leurs enfants et les faire grandir dans la religion catholique en disant à son mari qu'il valait mieux faire ce choix, "au cas où cela recommence ». Monsieur T. ne s'y est pas opposé.

Monsieur T. n'a pas raconté grand-chose de son histoire à ses enfants, et m'affirme qu'il était peu présent auprès de sa famille; c'est sa femme qui s'est occupée de l'éducation de leurs enfants. Ses trois enfants ont épousé des conjoints non-juifs. Une de ses petites-filles porte le prénom de sa grand-mère paternelle. Les relations avec ses enfants sont complexes, en particulier avec sa fille aînée. Ils «ne se comprennent pas».

Depuis le moment de la retraite, le temps psychique s'est modifié, et Monsieur T. pleure. Dans le même temps, depuis qu'il est à la retraite, soutenu par sa femme, il effectue des recherches sur son parcours d'enfant et sur celui de sa mère. Il a retrouvé des traces mais il y a également de nombreux trous. Monsieur T. dit qu'il est un homme qui a toujours su s'adapter aux situations difficiles.
Il aime continuer à réaliser des missions professionnelles à l'étranger, même bénévolement aujourd'hui. Mais il me dit porter des poids dont il n'arrive pas à se défaire.

Lors des séances, Monsieur T. pleure, en me parlant de lui mais surtout de son enfance, dont il a peu de souvenirs. Il a vécu l'Occupation entre l'âge de 2 et 5 ans.

Je propose à Monsieur T. de penser ses pleurs comme des sanglots qui se trouvent enfermés depuis sa petite enfance dans une «boite a-larme(s)». À 2 ans, séparé de sa mère, il n'a jamais pu pleurer sa perte, et il a espéré son retour. Rien ne lui a été dit. Lorsqu'il était caché, il ne pouvait pas évoquer la disparition de sa mère, il ne pouvait pas montrer sa tristesse car il aurait été en danger. Il a dû réprimer ses émotions, attendre qu'on vienne le chercher... Peut-être attend-il toujours? Alors les pleurs qui ne se sont pas manifestés, enfant, sont restés dans une boîte, bien fermée, car l'ouverture aurait été menaçante. Il s'est construit avec toutes ces larmes enfermées, toutes ces émotions emmurées et, en grandissant, cette boîte s'est verrouillée toujours un peu plus avec le temps. Après la guerre, le silence, l'exclusion de la parole s'est poursuivie. Un mur s'est construit en lui et la boîte a renforcé le verrouillage de sa fermeture, et toujours un peu plus. Les larmes sont restées ainsi enfermées car personne ne lui a jamais rien demandé.

La retraite est arrivée, le temps de l'emploi du temps s'est arrêté, un autre temps est advenu. Dans le processus de développement de l'enfant, la période de clandestinité a figé l'être, comme si l'horloge psychique de l'individu avait été arrêtée. Cet arrêt est la marque du traumatisme et a pour intention de reporter le deuil. À l'extérieur, dans la réalité, la reconnaissance de la souffrance des «enfants cachés» est advenue. En parallèle, Monsieur T. a lui-même ouvert un espace et un temps qu'il consacre à la recherche de traces de son enfance. L'appel est donc également venu du dedans. Alors cet appel a été tel que cette «boîtea-larme(s) » ne pouvait plus rester fermée, le loquet s'est ouvert et les larmes se sont (enfin) mises à s'écouler... Le signal d'alarme a été actionné et il ne pouvait plus le contrer.

Il apparaît que Monsieur T - comme d'autres personnes ayant vécu ce type d'expériences infantiles - se soit arrêté à une première étape du deuil, constituée du déni (Freud, 1915-1917) et d'une 
non-acceptation de la perte au niveau émotionnel (Bowlby, 1980). Endeuillé, Monsieur T. a évité toute occasion qui lui aurait rappelé la perte. Ce type de dysfonctionnement semble être fréquent chez les « enfants cachés » et a eu pour résultat l'arrêt partiel ou total du temps de croissance. Ainsi pourrait-on même affirmer que ce ne sont pas uniquement les sanglots qui ont été enfermés, mais c'est l'enfant lui-même qui l'a été. L'enfant a été muselé pendant toutes ces années. Tout ce que manifeste un enfant: l'expression de la vie, de l'imaginaire, les jeux, les émotions, le lien à l'objet primaire, ont été «confisqués », «emmurés». Et lorsqu'un fort appel ou des appels répétés à l'extérieur ou émanant du collectif se font entendre, le mur se fissure et laisse jaillir ce qui avait été mis de côté, ce qui avait été réprimé.

Dans ce suivi clinique, il a été question d'« empathie» (Lebovici, 2009; Golse, 2008), de bienveillance, de contenance telle qu'une mère se comporte avec son nourrisson. Cet homme a subi, enfant, une triple effraction: celle de son enveloppe psychique, celle de son enveloppe familiale, séparé brutalement de sa mère, de son père, de son inscription filiale et celle de son enveloppe culturelle, cible des attaques le mettant en danger de mort. Le processus thérapeutique a consisté à participer à « rétablir» ces enveloppes. Nous avons ensemble travaillé sur les représentations culturelles et notamment sa judaïté, son histoire et leur transmission à ses petits-enfants.

\section{En fin de vie, la re-naissance de Sarah}

Madame A. avait 72 ans quand elle est venue me voir. À la lecture d'un de mes articles, elle m'a fait part de sa satisfaction de voir qu'enfin quelqu'un savait parler de son histoire. Lors de son suivi, elle s'est souvenue s'être appelée Marie entre 1942 et 1945. Elle avait été baptisée catholique et avait toujours cru, depuis lors, se sentir plus attirée par le catholicisme que par le judaïsme. Or, au cours de ce travail en psychothérapie, elle a réalisé qu'elle se sentait davantage proche du judaïsme. Elle a ainsi pensé avoir été dans l'erreur pendant plusieurs décennies. Elle avait épousé un homme comme elle, juif qui avait été protégé par l'Eglise catholique pendant l'Occupation. Il avait été baptisé et avait fait sa première communion. Tous deux avaient tenté d'élever leurs enfants dans les deux religions, pensant qu'il était pertinent d'initier une nouvelle affiliation à partir de leurs deux affiliations : c'est ainsi qu'ils ont fait baptiser et circoncire leurs fils. Lors de ce suivi, Madame A. culpabilisait beaucoup de n'avoir pas compris le sens de son histoire et de sa construction. Elle se rendait compte de son échec auprès de ses enfants: ils étaient instables affectivement, ils étaient dans une grande dépendance visà-vis de leurs parents, incapables de s'autonomiser. À l'adolescence, l'un de ses fils avait fait une tentative de suicide, l'autre avait présenté des troubles psychologiques. J'ai suivi Madame A. durant deux années, puis j'ai poursuivi l'accompagnement en me rendant chez elle, jusqu'à quelques semaines avant qu'elle ne meure, atteinte d'un cancer. Elle a tenu à poursuivre un travail sur elle-même et c'est vers l'apaisement qu'elle s'en est allée. Nous avions pu évoquer ensemble les rituels de mort. Elle souhaitait être incinérée «pour rejoindre sa mère », morte à Auschwitz.

\section{Une enfance volée}

Les enfants juifs cachés ont été empêchés en tant qu'enfants de vivre «normalement", c'est-à-dire qu'ils ne pouvaient pas manifester un certain nombre d'émotions qu'exprime un enfant dans un environnement qui ne lui serait pas menaçant (Gampel, 1992). Leur enfance a été «volée»-pour reprendre le terme de Tomkiewicz (1999), caractérisant de cette façon son adolescence.

Ferenczi (1932) souligne le risque de dissociation entre une personnalité aux aspects matures et un aspect figé, bloqué dans l'enfance. Cet auteur ajoute que «si les chocs se succèdent au cours du développement, le nombre et la variété des fragments clivés s'accroissent et il nous devient rapidement difficile, sans tomber dans la confusion, de maintenir le contact avec les fragments, qui se comportent tous comme des personnalités distinctes qui ne se connaissent pas les unes des autres» (p. 133). C'est alors un état d'«atomisation ». C'est donc de clivage de la personnalité dont il s'agit. «Le trauma crée un clivage dans l'identité psychique, c'est un corps étranger flottant qui n'a pas sa place dans le système des représentations ordinairement communicables » (Doray, 2002, p. 106), et les enfants victimes ont appris trop tôt que les adultes peuvent eux-mêmes être clivés. Ils ont, précocement, rencontré une part obscure de l'être humain. Dasberg (2001) propose 
l'«Adult child survivor syndrome» pour caractériser la psychopathologie des adultes qui, enfants, ont survécu à la Shoah. Le trait caractéristique des adultes est le clivage, qui vient souligner un travail de deuil toujours reporté, jamais abouti.

Ces enfants ont mis en place des aménagements défensifs spécifiques pour survivre (Gampel, 1992), afin d'éviter tout risque ou "crainte d'effondrement»(Winnicott, non daté), pendant la guerre, au lendemain et depuis lors. Cependant des fragilités, telles des failles, subsistent. Il semble alors que ces fentes soient amenées à se révéler, à se dévoiler de façon plus importante en fin de vie et peut-être à la génération suivante (Feldman et Rottman, 2014).

Pour Fogelman (1991), la réunion qui s'est tenue à New-York en 1991 a été un événement sans précédent qui a permis d'enclencher un processus de guérison, notamment par la rupture de l'isolement. La prise en charge psychologique a consisté, à partir de ce moment-là, à permettre au groupe des «enfants cachés» de réaliser collectivement ce deuil. Le deuil individuel n'ayant pas pu se faire, c'est la reconnaissance d'un groupe ayant vécu cette même souffrance qui a permis l'enclenchement du processus de deuil.

\section{«Je suis un déchet»}

Lorsque je la reçois, Madame S. a 83 ans. Elle m'a été orientée par son psychiatre qui la suit depuis plusieurs années. À lâge de 10 ans, Madame S. a été cachée dans une poubelle du camp de concentration où elle et son père étaient internés - seul un nazi tolérait la présence de l'enfant, car son père travaillait dans l'atelier du camp et était doté d'une expertise dans le travail d'horlogerie, dont les nazis avaient besoin. Elle pouvait parfois aller dehors, dans la forêt, mais en ayant toujours la consigne de ne se montrer à personne. Cette histoire, elle ne comprend pas pourquoi elle me la raconte dès la première séance, plus de soixante-dix ans après l'avoir vécue, et c'est la première fois qu'elle en parle. Madame S. pleure beaucoup. Elle décrit des douleurs importantes dans son corps, dans toutes ses articulations. Elle a des difficultés à se mouvoir, à marcher, elle parle du fait que sa colonne vertébrale se tasse, elle a mal au dos. Après quelques séances, elle me dit: "C'est la guerre dans mon corps, comme celle que j'ai vécue». Lors d'une séance suivante, en parlant de son mal-être, de sa solitude, elle me dit: "Je suis une poubelle», puis elle dira: «Je suis un déchet».

Madame S. pleure, et à chaque début de séance elle dit qu'elle ne va pas bien. Elle a un traitement aux antidépresseurs, que son psychiatre réajuste régulièrement, mais rien n'y fait...

En décrivant ses douleurs, elle associe beaucoup avec le contexte actuel en France. Depuis les attaques meurtrières perpétrées dans les locaux de Charlie Hebdo et de l'Hypercacher les 7 et 9 janvier 2015, puis celles qui ont eu lieu en novembre de la même année, au Bataclan et dans les bistrots des $10^{\mathrm{e}}$ et $11^{\mathrm{e}}$ arrondissements de Paris, Madame S. a peur. Elle dit revivre ses peurs d'enfant: camouflée dans la poubelle, elle devait rester cachée, ne pas se montrer sinon elle risquait de mourir. Aujourd'hui, elle ne sort plus de chez elle, elle dit être effrayée par le monde extérieur.

La poubelle dans laquelle elle se trouvait semble être réactivée et peut-être constitue-t-elle un nouveau refuge aujourd'hui... Une question se pose: quelle représentation a-t-elle d'elle aujourd'hui?

\section{En conclusion, « une crainte de l'effondrement» réactivée par la réalité sociale}

Dans ce récit récent de Madame S., il apparaît donc qu'au-delà des passages de cycle de vie, qui sont sources de bouleversement, vient se manifester l'impact du collectif, de la réalité extérieure sur les éprouvés individuels.

La France a été un pays protecteur et, dans le même temps, menaçant. Durant toute leur vie, les enfants juifs cachés se sont construits selon un mode ambivalent avec ce pays, qui retentit sur leurs modalités d'attachement, en ballottage entre la protection et la menace. Au-delà de l'histoire d'une France coupée en deux pendant la guerre, collaborationniste et résistante, et de «Vichy, un passé qui ne passe pas» (Conan et Rousso, 1994), mentionnons de plus qu'entre 1967 et 1995, soit le temps d'une génération, une série d'événements est venue participer, réactiver, renforcer des éprouvés ambivalents: le discours de Ch. de Gaulle en 1967 sur les Juifs, «peuple d'élite, sûr de lui-même et dominateur»; en 1980, la phrase de Raymond Barre lors de l'attentat de la rue Copernic, qui fait 
une distinction parmi les victimes de l'attentat, des «Français innocents » à côté des «Israélites se rendant à la synagogue »; les années Mitterrand avec l'absence de reconnaissance des crimes commis par le gouvernement de Vichy, et le fleurissement annuel de la tombe de Pétain...

Bien que la question de partir, notamment vers Israël, se soit posée à la plupart d'entre eux à un moment de leur vie, peu parmi eux sont partis après la Libération, car le risque d'atteinte à leur sentiment de sécurité semblait alors à l'œuvre.

Ainsi, ces enfants, en grandissant et devenant adultes, n'ont pu tisser des liens stables, susceptibles d'assurer une sécurité intérieure permettant de se construire au mieux. Cette absence de sécurité interne est devenue un manque d'étayage psychique, et sans territoire sécurisant, et qui fait craindre un «risque d'effondrement» dans la dernière décennie de vie. Cette «crainte de l'effondrement» se lie à une faillite de l'organisation défensive face au vécu précoce que le Moi n'a pas pu intérioriser. Cet effondrement originel se retrouve alors sans trace, sans lien, sans temporalité. Il constitue un traumatisme psychique. Le traumatisme originel déborde le Moi et ne peut s'inscrire dans l'appareil psychique.

Le paradoxe d'avoir eu la chance de rester en vie est à l'origine de cette relation ambivalente qui aujourd'hui semble être renforcée par le contexte géopolitique actuel. Leurs inquiétudes sont portées sur eux-mêmes, mais surtout vers leurs enfants et leurs petits-enfants... Une peur «que cela recommence».

\footnotetext{
The question of early trauma being re-enacted through the breaches of current day events in society Oummary is raised. We relate here the stories of jewish children hidden away in France during the Second World War. How did they manage through the different stages of psychic restructuration during their lives: adolescence, parenting, grandparenting, retirement? Three clinical cases are discussed: going on retirement for one patient, the approach of death for another and thirdly the murderous attacks of January 2015 in France for another. These events reenact what was never able to be worked through from the early part of these patients' lives and how, and show necessary it is to do so with hundsight even at an advanced age.
}

\section{Bibliographie}

Altounian J. (2000): La survivance. Paris, Dunod.

Bowlby J. (1980): Attachement et perte: III. La perte, tristesse et dépression. Paris, PUF.

Cherki A. (2006): La frontière invisible. Paris, Elema.

Conan E., Rousso H. (1994): Vichy, un passé qui ne passe pas. Paris, Fayard.

Dasberg H. (2001) Adult child survivor syndrome. One deprived childhood of aging Holocaust survivors. Israel Journal of Psychiatry and Related Sciences, 38 (1): 13-26.

Doray B. (2002): La trace d'un traumatisme dans un récit de Georges Pérec, in: Bertrand M. : Les enfants dans la guerre et les violences civiles. Approches cliniques et théoriques (pp. 105-116). Paris, l'Harmattan.

Dwork D. (1991): Children With A Star: Jewish Youth in Nazi Europe. New York, VailBallou Press.

Feldman M. (2009): Entre trauma et protection: quel devenir pour les enfants juifs cachés en France (1940-1944)? Toulouse, Erès.

Feldman M. (2016): Sauvés mais "exposés". Impacts des traumas d'enfance tardivement reconnus. A propos des enfants juifs cachés en France pendant la Seconde Guerre Mondiale, in: Les enfants exposés aux violences collectives (pp. 59-71). Toulouse, Erès.
Feldman M., Hammami S. (2017): Une expérience clinique auprès d'aidants de patients atteints de la maladie d'Alzheimer survivants de la Shoah: un soin auprès de patients «doublement cachés». Dialogue, 216: 53-66.

Feldman M., Mansouri M., Moro M.R. (2013): Être une adolescente juive pendant l'Occupation en France: quel devenir d'adulte? Adolescence, 31 (3): 601-612.

Feldman M., Mouchenik Y., Moro M.R. (2008): Les enfants juifs cachés en France pendant la Seconde Guerre mondiale: des traces du traumatisme repérables plus de soixante ans après, La Psychiatrie de l'Enfant, 51 (2): 481-513.

Feldman M., Rottman H. (2014): Du vécu au perçu: à propos des enfants juifs cachés en France pendant l'Occupation et de la descendance, in: Une génération, l'autre, sous la direction de Bantman P., Paris, In Press, 119-134.

Ferenczi S. (1932). La confusion des langues, in: CEuvres complètes, tome 4 -1927-1933 (pp. 125-147). Paris, Payot, 1990.

Fogelman E. (1991): A Psychology Behind a Hidden Child, in: Marks J.: The Hidden Children (pp. 292-307). New York, Ballantine Books, 1993.

Freud S. (1915): Deuil et mélancolie, in: Métapsychologie (pp. 43-76). Paris, Payot, 2010. 
Gampel Y. (1992): I was a Shoah Child. British Journal of Psychotherapy, 8 (4): 391-400.

Golse B. (2008): Du bébé observé au bébé soigné: la place de l'empathie. La Psychiatrie de l'Enfant, 51 (2): 325-337.

Hazan K. (2000): Les orphelins de la Shoah. Les maisons de l'espoir. Paris, Belles Lettres.

Khan M. (1976): Le soi caché. Paris, Gallimard.

Lebovici S. (2009): L'empathie, in: L'arbre de vie. Eléments de la psychopathologie du bébé (pp. 101-111). Toulouse, Erès.

Tomkiewicz S. (1999) : L'adolescence volée, Paris, Calmann-Lévy.

Winnicott D.W. (non daté): La crainte de l'effondrement, in: La crainte de l'effondrement et autres situations cliniques (pp. 205-216). Paris, Gallimard, 2015.

Winnicott D.W. (1956): La préoccupation maternelle primaire, in: De la pédiatrie la psychanalyse (pp. 285-291). Paris, Payot, 1992.
Correspondance

Marion Feldman

Université Paris Nanterre

200 avenue de la République

92001 Nanterre cedex

France

Courriel: m.feldman@parisnanterre.fr 